\title{
DIAGNÓSTICO DE SINÉQUIAS UTERINAS POR HISTEROSSONOGRAFIA TRANSVAGINAL
}

Hélio Antonio Guimarães Filho*, Rosiane Mattar, Edward Araujo Júnior, Cláudio Rodrigues Pires, Antonio Fernandes Moron

Trabalho realizado no Departamento de Obstetrícia da Universidade Federal de São Paulo (Unifesp-EPM), São Paulo, SP

\author{
*Correspondência: \\ Rua Reinaldo Tavares de Melo, 142 \\ Apto. 901, Manaíra \\ CEP 58038-300 \\ João Pessoa, PB \\ helioagf@uol.com.br
}

\begin{abstract}
RESUMO
OBjetivo. Avaliar a prevalência de sinéquias uterinas em pacientes com aborto recorrente e a acurácia diagnóstica da ultrasonografia transvaginal (US-TV) e da histerossonografia (HS).

Métodos Sessenta pacientes não grávidas com passado de três ou mais abortos espontâneos consecutivos foram avaliadas por US-TV, HS e histeroscopia (HTC) para a pesquisa de sinéquias uterinas. A HTC foi considerada o padrão ouro do estudo. A concordância dos achados da US-TV e da HS foram avaliadas pelo coeficiente Kappa e sua significância foi testada. O nível de significância adotado foi de $0,05(\alpha=5 \%)$. Foram calculadas as medidas de sensibilidade, especificidade e valor preditivo positivo e negativo para cada um dos métodos.
\end{abstract}

Resultados. Observou-se a presença de sinéquias uterinas em 16 (26,7\%) pacientes. A acurácia da US-TV e da HS foram, respectivamente, de 78,9\% e 92,7\%. Comparativamente à US-TV, a HS foi muito superior quanto à sensibilidade $(78,6 \%$ versus $20 \%$ ) e concordância com a HTC (Kappa $=80 \%$ versus Kappa $=27 \%)$.

ConcLusão. Observou-se boa concordância da HS e concordância ruim da US-TV em relação à HTC para o diagnóstico de sinéquias uterinas. Devido à sua baixa sensibilidade, a US-TV não demonstrou ser um método aplicável à investigação de sinéquias uterinas em pacientes com aborto recorrente. A HS, por sua vez, parece oferecer importante contribuição nesta pesquisa, particularmente por sua simplicidade técnica, baixo custo e elevada acurácia diagnóstica.

UnItermos: Aborto recorrente. Sinéquia. Ultra-sonografia. Histerossonografia.

\section{INTRODUÇÃO}

As alterações da cavidade uterina têm prevalência significativamente elevada em pacientes com passado de abortamento habitual, sendo relatada entre $15 \%$ até $50 \%$ dos casos'. Embora ainda permaneça controvertida a relação direta de causa e efeito entre alguns tipos de alterações da cavidade uterina com a perda gestacional de repetição, existem evidências suficientes de que a correção cirúrgica de determinados defeitos, incluindo as sinéquias intra-uterinas, podem determinar melhora significativa no prognóstico reprodutivo destas pacientes ${ }^{2,3}$.

Sinéquias são aderências intra-uterinas geralmente resultantes de endometrites, curetagens, cirurgia intra-uterina para remoção de mioma ou correção de defeito estrutural do útero e operação cesareana². Consiste, portanto, em uma anormalidade uterina adquirida, sendo considerada responsável por alterações menstruais, infertilidade e abortamento habitual2,3. Assim, um aborto pode ocorrer como conseqüência de redução da superfície endometrial para implantação embrionária e pela dificuldade de expansão da cavidade uterina ${ }^{4,5}$.

As sinéquias podem ser classificadas em três estágios: aderências leves, que são membranas compostas por tecido endometrial, podendo ser parciais ou totais; aderências moderadas, formadas por tecido fibromuscular ainda revestido por endométrio, caracteristicamente espessas, que podem ocluir a cavidade uterina de forma parcial ou total; e aderências severas, que ocluem parcial ou totalmente a cavidade, compostas apenas por tecido conectivo denso ${ }^{5}$. $O$ prognóstico reprodutivo é pior quanto mais severa for a adesão, sendo importante também a sua localização, $0^{6,7}$.
Tradicionalmente, as sinéquias uterinas têm sido pesquisadas por meio da ultra-sonografia transvaginal (US-TV), histerossalpingografia (HSG) e histeroscopia diagnóstica (HTC) $)^{8}$. A histerossonografia (HS), por sua vez, surgiu como técnica simples, de baixo custo e bem tolerada, capaz de refinar o diagnóstico ultra-sonográfico das patologias uterinas intra-cavitárias, sendo que diversos estudos têm demonstrado sensibilidade e especificidade comparáveis à HTC 9,10,11,12. Ao considerar que uma rotina propedêutica por vezes não uniformizada, invasiva e desconfortável tem sido proposta para o estudo das anormalidades uterinas em pacientes com AER, a HS, diante de suas vantagens, parece ser técnica interessante na investigação de alterações uterinas intracavitárias neste grupo de pacientes.

O presente estudo teve como objetivo pesquisar a prevalência de sinéquias uterinas numa população de pacientes com abortamento habitual, por meio da US-TV e da HS, e avaliar a acurácia destes métodos para o diagnóstico desta alteração.

\section{Métodos}

Realizou-se estudo transversal do tipo descritivo. A amostra foi constituída de 60 pacientes atendidas no ambulatório de abortamento habitual da Universidade Federal de São Paulo - Escola Paulista de Medicina (Unifesp/EPM), entre março de 2003 e dezembro de 2004. Foram incluídas no estudo pacientes não grávidas com três ou mais abortos recorrentes consecutivos. Os critérios de exclusão compreenderam pacientes com infecção pélvica aguda ou recente, patologia da cavidade uterina previamente conhecida pelos examinadores e 


\section{Tabela I - Sensibilidade, especificidade, VPP, VPN, acurácia diagnóstica e concordância com o padrão ouro} da US-TV e HS para o diagnóstico de sinéquias

\begin{tabular}{|c|c|c|c|c|c|c|}
\hline Sinéquias & Sensibilidade & Especificidade & VPP & VPN & Acurácia & Concordância \\
\hline US-TV & $\begin{array}{c}3 / 15 \\
(20,0 \%)\end{array}$ & $\begin{array}{c}42 / 42 \\
(100,0 \%)\end{array}$ & $\begin{array}{c}3 \beta \\
(100,0 \%)\end{array}$ & $\begin{array}{c}42 / 54 \\
(77,8 \%)\end{array}$ & $\begin{array}{c}45 / 57 \\
(78,9 \%)\end{array}$ & $\begin{array}{c}\text { Kappa }=27 \% \\
p=0,003\end{array}$ \\
\hline HS & $\begin{array}{c}11 / 14 \\
(78,6 \%)\end{array}$ & $\begin{array}{c}40 / 41 \\
(97,6 \%)\end{array}$ & $\begin{array}{c}11 / 12 \\
(91,7 \%)\end{array}$ & $\begin{array}{c}40 / 43 \\
(93,0 \%)\end{array}$ & $\begin{array}{c}51 / 55 \\
(92,7 \%)\end{array}$ & $\begin{array}{c}\text { Kappa }=80 \% \\
p<0,001\end{array}$ \\
\hline
\end{tabular}

Os resultados são expressos em números de pacientes, com a porcentagem entre parênteses. VPP, valor preditivo positivo. VPN, valor preditivo negativo. US-TV, ultra-sonografia transvaginal. HS, histerossonografia

gravidez suspeita ou confirmada. O estudo foi aprovado pelo Comitê de Ética em Pesquisa da Unifesp-EPM.

As pacientes assinaram termo de consentimento esclarecido e foram submetidas à avaliação da cavidade uterina por meio de US-TV, HS e HTC.

Inicialmente, todas as pacientes foram submetidas a US-TV, que foi realizada na segunda fase do ciclo menstrual. Foi utilizado aparelho de ultra-sonografia da marca Toshiba Power Vision 6000, com transdutor endocavitário multifreqüencial de $4,2 \mathrm{MHz}$ a $7,5 \mathrm{MHz}$. Imagens caracterizadas por bandas hipoecogênicas cruzando 0 endométrio hiperecóico ou interrupções do eco endometrial que aparecem em continuidade com o miométrio em pelo menos uma incidência foram consideradas como sinéquias.

Na seqüência, desta vez na primeira fase do ciclo menstrual, realizou-se a HS. Para a cateterização do colo uterino, utilizou-se sonda Foley siliconizada $n^{\circ} 6$, com balão inflável em sua extremidade. A infusão de solução salina estéril foi feita a partir de seringa de $20 \mathrm{ml}$ acoplada ao cateter, sob visão ecográfica em tempo real da cavidade uterina. Imagens observadas como separação incompleta dos folhetos endometriais anterior e posterior durante a instilação salina, caracterizada por bandas ecogênicas finas ou espessas que cruzam a cavidade, foram diagnosticadas como sinéquias. Os exames de US-TV e HS foram executados por um mesmo examinador.

Finalmente, as pacientes foram submetidas a HTC diagnóstica na primeira fase do ciclo. Para tanto, foi utilizado vídeo-histeroscópio rígido de 2,9 mm, acoplado a fonte de luz de Xenon com 150 Watts. Neste exame, foram consideradas como sinéquias a presença de faixas irregulares de tecido que atravessam a cavidade endometrial. O médico que realizou os exames de HTC não tinha conhecimento dos resultados da US-TV e da HS.

Os achados obtidos em cada método foram tabulados e agrupados entre si quanto à presença de sinéquias. Foi avaliada a sua freqüência e os métodos foram comparados. A HTC foi considerada neste estudo como o padrãoouro.

A concordância dos achados da US-TV e da HS em relação à HTC foi avaliada pelo coeficiente Kappa e sua significância foi testada. $O$ coeficiente Kappa quantifica a concordância entre dois métodos realizados nos mesmos indivíduos, sendo assim interpretados: $81 \%$ a $100 \%$ (ótima); $61 \%$ a 80\% (boa); $41 \%$ a 60\% (regular); 21\% a 40\% (ruim) e menor do que $20 \%$ (péssima) $^{13}$. Foram calculadas as medidas de sensibilidade, especificidade, valores preditivo positivo (VPP) e negativo (VPN) e acurácia da US-TV e da HS em relação ao resultado da HTC. O nível de significância adotado foi de $0,05 \%(\alpha=5 \%)$.

\section{Resultados}

Considerando os exames US-TV e HTC, tem-se:

- Em dois pacientes a HTC foi inconclusiva ou falha, e a US-TV foi normal;

- Em um paciente a US-TV foi inconclusiva ou falha, e a HTC foi alterada.

Dessa forma, 57 mulheres apresentaram resultados confiáveis em ambos os exames.

Considerando os métodos HS e HTC, obteve-se:

- Em dois pacientes a HTC foi inconclusiva ou houve falha do método, e a HS foi normal em uma delas e alterada na outra;

- Em três pacientes a HS foi inconclusiva ou houve falha do método, e a HTC foi normal em uma delas e alterada nas outras duas pacientes.

Dessa forma, 55 mulheres apresentaram resultados confiáveis em ambos os exames.

Com base nesta amostra, foi observada a presença de sinéquias em I 6 (26,7\%) pacientes por meio da HTC, I3 (21,7\%) por meio da HS, e $3(5 \%)$ por meio da US-TV. A concordância entre os exames de USTV e HTC foi considerada ruim na detecção da presença de sinéquias (Kappa $=27 \%)$, enquanto a concordância entre os exames de HS e HTC foi considerada boa (Kappa $=80 \%$ ). Estes resultados, incluindo os valores de sensibilidade, especificidade, valores preditivos positivo e negativo e acurácia diagnóstica, estão representados na Tabela I.

\section{Discussão}

A presença de sinéquias uterinas está relacionada com diversas situações clínicas na prática gineco-obstétrica. Em pesquisa realizada no ambulatório de abortamento habitual da UnifespEPM no ano de 2004, foi encontrada uma prevalência de $21,6 \%$ para esta alteração, em pacientes abortadoras habituais ${ }^{14}$. No presente estudo, encontramos uma prevalência de 26,7\% para sinéquias neste grupo de pacientes, resultado este semelhante aos relatados da literatura ${ }^{14,15}$. Entretanto, ainda há controvérsias sobre a importância de um defeito desta natureza na gênese de um estado de infertilidade ou de uma perda gestacional, bem como da gravidade do defeito necessária para a ocorrência de tais 
desfechos adversos ${ }^{16}$. Todavia, a sua pesquisa, particularmente nestas situações clínicas descritas, tem sido sistematicamente realizada.

A HTC, embora reconhecida como o método mais confiável na avaliação endometrial, tem a considerar o custo do material e do treinamento de pessoal qualificado, bem como o tempo dispensado na esterilização e uso do histeroscópio, fatores que são potencialmente limitantes para sua utilização rotineira, principalmente em serviços com grande demanda de pacientes.

A US-TV, devido ao seu fácil acesso e baixo custo, tem sido o exame inicial na investigação dos órgãos genitais internos femininos, incluindo-se o estudo da arquitetura endometrial ${ }^{10}$. Todavia, a despeito da evolução dos equipamentos e melhoria acentuada na resolução das imagens obtidas por meio de transdutores de alta frequêência, alguns trabalhos têm chamado a atenção para sua reduzida acurácia no diagnóstico de sinéquias uterinas ${ }^{11,17,18}$. No presente estudo, demonstrou-se a pobre caracterização desta alteração pela US-TV (concordância ruim; Kappa $=27 \%$ ), sendo esta detectada em apenas $5 \%$ dos casos (sensibilidade de apenas 20\%), quando comparada aos resultados da HTC. Estes resultados obtidos estão próximos aos encontrados na literatura ${ }^{11,17,18}$. Em um dos casos identificados pela US-TV, observouse a presença de apenas uma área suspeita, enquanto que após a distensão da cavidade pela solução salina verificou-se que duas áreas de adesão estavam presentes; achados confirmados pela HTC.

Soares et al." verificaram péssima acurácia da US-TV para o diagnóstico de sinéquias uterinas. Em seu estudo, a US-TV não foi capaz de identificar nenhuma das quatro adesões diagnosticadas pela HTC (sensibilidade e VPP de 0\%).

Romer et al. ${ }^{17}$, comparando os achados da HTC e da US-TV para o diagnóstico de sinéquias, observaram que a US-TV diagnosticou corretamente apenas 33\% dos casos.

Há um único estudo em que foi observada excelente acurácia da US-TV para o diagnóstico de sinéquias. Fedele et al. ${ }^{19}$ encontraram sensibilidade de $91 \%$ e especificidade de 100\% da US-TV para a detecção de adesões intracavitárias, com VPP e VPN de 100\% e $98,5 \%$, respectivamente. Tais resultados, todavia, jamais conseguiram ser reproduzidos por diversas pesquisas que se seguiram ${ }^{11,17,18,20}$.

Com relação à $H S$, vários estudos já demonstraram elevada acurácia do método para a detecção e graduação da severidade das sinéquias uterinas, ${ }^{|, 42|}$.

Accorsi Neto et al. ${ }^{21}$ realizaram estudo comparativo em que foram avaliadas 58 mulheres na pós-menopausa por meio de HS e HTC. Esses autores observaram elevada concordância dos achados de sinéquia uterina entre os dois métodos. Foram diagnosticadas II sinéquias por meio da HS e 12 por meio da HTC diagnóstica, correspondendo, respectivamente, a 21\% e 24\% de todas as alterações intracavitárias observadas por cada um dos métodos.

Gaucherand et al. ${ }^{22}$ avaliaram por HS 19 pacientes com história de abortamento recorrente ou amenorréia secundária, e observaram sensibilidade de 100\% para o diagnóstico de sinéquias.

A US-TV, pelo seu fácil acesso e baixo custo, é quase que invariavelmente o primeiro exame realizado na investigação do fator uterino em pacientes com abortamento habitual. Todavia, possui acurácia diagnóstica limitada, o que requer, na maioria das vezes, prosseguimento na investigação por meio de exame que possibilite a distensão da cavidade uterina em busca de alterações que são difíceis de ser detectadas pelo exame ultra-sonográfico convencional, tais como as sinéquias. A complementação da US-TV com a HS preenche esta exigência propedêutica, possibilitando adequada avaliação da paciente em um único momento, simplificando a investigação. Neste estudo, o elevado VPN encontrado para a HS (93\%) sugere que a avaliação em visita única (US-TV seguida de HS) pode ser uma alternativa bastante interessante para este grupo de pacientes, reduzindo o seu desconforto e os custos com exames desnecessários, com esterilização de material e com honorários médicos, bem como diminuindo o número de faltas ao trabalho devido a visitas consecutivas (realização sistemática de US-TV, HSG e/ou HTC).

De fato, diversos autores têm concordado que a HS é método diagnóstico de execução mais fácil, mais acessível, menos dispendioso, mais seguro, menos invasivo e mais bem tolerado do que a HSG e HTC diagnóstica, com acurácia similar a esta última 7.10,23. Os resultados do presente estudo demonstraram boa concordância da HS (sensibilidade de 78,6\%; Kappa = 80\%) com a HTC para a caracterização das adesões intra-uterinas, sendo muito superior à US-TV em definir corretamente a presença, localização, quantidade, espessura e extensão das mesmas. A concordância geral entre a HS e a HTC, englobando os resultados verdadeiro-positivos e verdadeiro-negativos, foi de $92,7 \%$, indicando que a HS pode seguramente substituir a HTC numa elevada proporção de casos, com suas respectivas vantagens já discutidas.

\section{ConClusão}

Os resultados aqui obtidos sugerem que a HS pode ser alternativa de baixo custo e de elevada acurácia, refinando a investigação ultra-sonográfica da cavidade uterina em pacientes com abortamento espontâneo de repetição, proporcionando importante contribuição no diagnóstico de sinéquias intra-uterinas neste grupo de pacientes.

\section{Conflito de interesse: não há}

\section{SUMMARY}

\section{Diagnosis OF UTERINe SYNechiae IN PATIENTS WITH RECURRENT MISCARRIAGES: CONTRIBUTION OF TRANSVAGINAL HYSTEROSONOGRAPHY}

BACKGROUND. The aim of this study was to determine the prevalence of uterine synechiae in patients with recurrent miscarriages and to evaluate the diagnostic accuracy of transvaginal ultrasound and of hysterosonography.

METHODS. Sixty non-pregnant patients with a history of at least three previous consecutive miscarriages were evaluated by transvaginal ultrasound, hysterosonography and hysteroscopy to detect uterine synechiae. Hysteroscopy was considered the gold standard. Agreement of findings disclosed by transvaginal ultrasound and by the hysterosonography were evaluated according to the Kappa coefficient and their significance was tested. Significance was established at $<0$. 05 (Alpha error $=5 \%$ ). Sensitivity, specificity, positive and negative predictive values were determined for each method. 
RESULTS. Uterine synechiae were identified in 16 patients (26.7\%). The accuracy of the transvaginal ultrasound and of the hysterosonography was $78.9 \%$ and $92.7 \%$, respectively. When compared to the transvaginal ultrasound, hysterosonography had a much greater sensitivity (78.\% vs. 20.0\%) and a higher degree of agreement with hysteroscopy (Kappa = $80 \%$ vs. Kappa $=27 \%$ ).

CONCLUSION. For diagnosis of uterine synechiae, hysterosonography had a higher level of agreement with hysteroscopy than the transvaginal ultrasound. In patients with recurrent miscarriages transvagina/ ultrasound is not recommended for the investigation of uterine synechiae because of its low sensitivity. Hysterosonography, on the other hand, seems to offeran important contribution especially because it is a simple, low-cost and accurate method for diagnosis of uterine synechiae. [Rev Assoc Med Bras 2006; 52(5): 308-II]

KEY WORDS: Recurrent miscarriage. Synechiae. Ultrasound. Hysterosonography.

\section{REFERÊNCIAS}

I. Keltz MD, Olive DL, Kim AH, Arici A. Sonohysterography for screening in recurrent pregnancy loss. Fertil Steril 1997;67(4):670-4.

2. Schenker JG, Margalioth EJ. Intrauterine adhesions: an update appraisal. Fertil Steril 1982;37(5):593-6 I0.

3. Valle RF, Sciarra JJ. Intrauterine adhesions: hysteroscopic diagnosis, classification, treatment, and reproductive outcome. Am J Obstet Gynecol 1988; I 58(| 4): | 459-66.

4. Salle B, Gaucherand P, de Saint Hilaire P, Rudigoz RC. Transvaginal sonohysterography evaluation of intrauterine adhesions. J Clin Ultrasound 1999;27(3): 131-4.

5. Patton PE. Anatomic uterine defects. Clin Obstet Gynecol 1994:37(3):705-21.

6. Goldenberg M, Sivan E, Sharabi Z, Mashiach S, Lipitz S, Seidman DS. Reproductive outcome following hysteroscopic management of intrauterine septum and adhesions. Hum Reprod 1995; I 0( I 0):2263-5.

7. The American Fertility Society. The American Fertility Society classifications of adnexal adhesions, distal tubal occlusion, tubal occlusion secondary to tubal ligation, tubal pregnancies, mullerian anomalies and intrauterine adhesions. Fertil Steril 1988;49(6):944-55.

8. Brown SE, Coddington CC, Schnorr J, Toner JP, Gibbons W, Oehninger S. Evaluation of outpacient hysteroscopy, saline infusion histerosonography, and hysterosalpingography in infertile women: a prospective, randomized study. Fertil Steril 2000;74(5): 1029-34.

9. Widrich T, Bradley LD, Mitchinson AR, Collins RL. Comparison of saline infusion sonography with office hysteroscopy for the evaluation of the endometrium. Am J Obstet Gynecol 1996; 1 74( I I): 1 327-34.
10. Gronlund L, Hertz J, Helm P, Colov NP. Transvaginal sonohysterography and hysteroscopy in the evaluation of female infertility, habitual abortion or metrorrhagia. A comparative study. Acta Obstet Gynecol Scand 1999;78(4):415-8.

I I. Soares SR, Barbosa dos Reis RR, Camargos AF. Diagnostic accuracy of sonohysterography, transvaginal sonography, and hysterosalpingography in pacients with uterine cavity diseases. Fertil Steril 2000;73(2):406-II .

12. Laifer-Narin SI, Ragavendra N, Parmenter EK, Grant E. False-normal appearance of the endometrium on conventional transvaginal sonography comparison with saline hysterosonography. Am J Roentgenol 2002; 178(1): 129-33.

13. Altman DG. Practical statistics for medical research. Londres: Chapman \& Hall/CRC; 1991.

14. Traina E, Mattar R, Moron AF, Albuquerque Neto LC, Matheus ED. Acurácia diagnóstica da histerossalpingografia e da ultra-sonografia para avaliação de doenças da cavidade uterina em pacientes com abortamento recorrente. Rev Bras Ginecol Obstet 2004;26(7):527-33.

15. Raziel A, Arieli S, Bukovsky I, Caspi E, Golan A. Investigation of the uterine cavity in recurrent aborters. Fertil Steril 1994; 62(5): 1080-2.

16. Stirrat GM. Recurrent miscarriage I: definition and epidemiology. Lancet 1990;90(2):673-5.

17. Romer T, Bojahr B, Lober R, Grabow D. The investigation of the uterine cavity in infertility: comparison of three diagnostic methods. J Assisted Reprod Genet 1993; 10(6):201.

18. Shalev J, Meizner I, Bar-Hava I, Duker D, Mashiach R, Ben-Rafael Z. Predictive calue of transvaginal sonography performed before routine diagnostic hysteroscopy for evaluation of infertility. Fertil Steril 2000;73(2):4I2-7.

19. Fedele L; Bianchi S; Dorda M; Vignali M. Intrauterine adhesions: detection with transvaginal US. Radiology 1996; 199(3):757-9.

20. Loverro G, Nappi L, Vicino M, Carriero C, Vimercati A, Selvaggi L. Uterine cavity assessment in infertily women: comparison of transvaginal sonography and hysteroscopy. Eur J Obstet Gynecol Reprod Biol 2001; 100(1):67-71.

21. Accorsi Neto AC, Gonçalves WJ, Mancini SN. Comparação entre a histerossonografia, a histeroscopia e a histopatologia na avaliação da cavidade uterina de mulheres na pós-menopausa. Rev Bras Ginecol Obstet 2003;25(9):667-72.

22. Gaucherand P, Piacenza JM, Salle B, Rudigoz RC. Sonohysterography of the uterine cavity: preliminary investigations. J Clin Ultrasound 1995;23(3):339-48.

23. Valenzano MM, Lijoi D, Mistrangelo E, Fortunato T, Costantini S, Ragni N. The value of sonohysterography in detecting intracavitary benign abnormalities. Arch Gynecol Obstet 2005;6(I): I -4.

Artigo recebido: 16/09/2005

Aceito para publicação: 22/04/2006 